\title{
Relación entre violencia familiar y autoconcepto del educando, comunidad awajún de Tayuntsa, Amazonas, Perú
}

\author{
Relationship between family violence and the student's self-concept, Awajún \\ community of Tayuntsa, Amazonas, Peru
}

\author{
Mary Denisse Calvay Gomez ${ }^{1}$, Ermerith Kiak Timias ${ }^{2}$
}

\section{RESUMEN}

La investigación tuvo por objetivo determinar el grado de relación entre violencia familiar y autoconcepto del educando de sexto grado en la Institución Educativa Primaria $\mathrm{N}^{\circ} 16309$ de la comunidad awajún Tayuntsa, distrito de Nieva, provincia de Condorcanqui, departamento de Amazonas, Perú en el año 2017; la población muestral estuvo conformada por 24 padres de familia y 24 estudiantes. El estudio fue descriptivo y correlacional, utilizó como instrumento dos cuestionarios para evaluar la violencia familiar y escala de autoconcepto (Piers-Harris), válidos y confiables estadísticamente. Como resultados se encontró que el nivel de violencia familiar es moderadaalta (54\%) y el nivel de autoconcepto es deficiente-pobre (75\%) en el educando del sexto grado; resultados similares presentan las dimensiones de ambas variables. Finalmente se concluye que existe una correlación moderada negativa $(\mathrm{R}=-0.518, \mathrm{P}=0.029<0.05)$ entre la violencia familiar y el autoconcepto del educando del sexto grado en la Institución Educativa Primaria N. 16309 de la comunidad awajún Tayuntsa.

Palabras clave: Autoconcepto, educandos, violencia familiar

\begin{abstract}
The objective of the research was to determine the degree of relationship between family violence and self-concept of the sixth grade student in the Primary Educational Institution No. 16309 of the Tayuntsa Awajún community, Nieva district, Condorcanqui province, Amazonas department, Peru in 2017; the sample population was made up of 24 parents and 24 students. The study was descriptive and correlational, using two questionnaires to assess family violence and a statistically valid and reliable self-concept scale (Piers-Harris) as an instrument. As results, it was found that the level of family violence is moderate-high (54\%) and the level of self-concept is deficient-poor (75\%) in the sixth grade student; similar results present the dimensions of both variables. Finally, it is concluded that there is a moderate negative correlation $(\mathrm{R}=-0.518, \mathrm{P}=0.029<0.05)$ between family violence and the selfconcept of the sixth grade student in the Primary Educational Institution No. 16309 of the Tayuntsa Awajún community.
\end{abstract}

Keywords: Self-concept, learners, family violence

\footnotetext{
Bachiller en Educación. Escuela Profesional de Educación Intercultural Bilingüe, Facultad de Educación y Ciencias de la Comunicación de la Universidad Nacional Toribio Rodríguez de Mendoza de Amazonas. Correo electrónico: 011116A121@untrm.edu.pe

${ }^{2}$ Bachiller en Educación. Escuela Profesional de Educación Intercultural Bilingüe, Facultad de Educación y Ciencias de la Comunicación de la Universidad Nacional Toribio Rodríguez de Mendoza de Amazonas. Correo electrónico: 011123A121@untrm.edu.pe
} 


\section{INTRODUCCIÓN}

La violencia familiar puede presentarse en diferentes formas y pasar desapercibida socialmente. Lo común es encontrar violencia familiar en sus variantes física, psicológica, económica y sexual; sin embargo, estas formas pueden también repercutir como violencia hacia los niños, hacia los mayores, entre parejas de esposos, a las mujeres e inclusive contra los discapacitados. Muchos estudios determinan que la violencia puede ocurrir en todas las clases sociales y en diferentes edades, aunque la mayoría de las veces se trata de los adultos hacia uno o varios individuos de menor edad, tal como demuestran los estudios de Patró y Limaña (2005) que las victimas más usuales terminan siendo las personas más débiles, en la mayoría de casos los hijos y la esposa. La Organización Mundial de la Salud - OMS (2009) pone en juego que el $10 \%$ y el $52 \%$ de mujeres, han sido víctimas de maltrato físico por parte de su esposo en algún momento de su vida y el 10\% y el $30 \%$ sufrió violencia sexual; declara, además, que el $10 \%$ y el $27 \%$ de las mujeres ha sido objeto de abusos sexuales, siendo niñas o adultas.

De igual manera, un colectivo de estudios realizados por Annerback, Wingren y Gutafsson (2010); Dubowitz y Benett (2007); Jouriles, McDonald, Smith y Heyman (2008) determinan que la violencia dada en el hogar hacia la pareja, incrementa el riesgo de violencia contra niños y adolescentes. Por otro lado, la violencia familiar contra niños, niñas y adolescentes, según la Encuesta Demográfica y de Salud Familiar -ENDES (2013), realizada en el Perú, revela que el $17.6 \%$ de las mujeres entrevistadas piensa que el castigo físico es necesario para educar a los hijos. Por su parte, Alcázar y Ocampo (2016) indican que la violencia familiar, desconcierta todo el ámbito familiar y vulnera el desarrollo integral de los niños y las niñas.

El Instituto Nacional de Estadística e Informática (2017), en adelante INEI, mostró cifras alarmantes para la región Amazonas, entre ellas se verifica que el $71,8 \%$ de las mujeres sufrieron algún tipo de violencia por parte de su pareja, destacando la violencia psicológica $y / 0$ verbal en un $65,8 \%$, la violencia física en un $37,9 \%$ y sexual en un $6,5 \%$. Estos datos son alarmantes y coincidentes con las estadísticas nacionales y mundiales. Así, por ejemplo, Orna (2013) sobre los tipos de violencia familiar según distritos de Lima Metropolitana, encontró que, en todos los distritos, el tipo de violencia familiar más predominante es la violencia física seguida de la psicológica. Por otro lado, el INEI (2017) según ámbito geográfico nos muestra que en la Sierra y Selva existe un $74,1 \%$ en promedio de violencia y en los departamentos de Apurímac $(85,1 \%)$, Huancavelica $(82,5 \%)$ y Cusco $(81,1 \%)$.

Por otra parte, si la violencia se genera en el hogar, el niño es partícipe de tal acontecimiento, como ya se mencionó anteriormente, bien puede ser víctima, es decir, ser el sujeto que recibe la acción o simplemente observador en otros de las prácticas de violencia. En esa lógica, el autoconcepto del niño se elabora constantemente, dependiendo de las circunstancias y de las relaciones mantenidas por el individuo, así como de la importancia que les asigne a las interacciones que establece (Goñi y Fernández, 2008). Si el autoconcepto de la persona está sujeto a las circunstancias, entonces la violencia familiar puede ser un factor influyente en este y termina también repercutiendo en la conducta del niño y en su rendimiento académico. En ese sentido, Bernal y Gálvez (2017) determinan que el concepto de sí mismo que posea una persona repercute enormemente no solo en su desempeño, sino también en el desarrollo y la estructuración de su personalidad. Desde esta perspectiva, la presente investigación, nos permitirá comprender el grado de asociación entre la violencia familiar y el autoconcepto del educando desde el nivel escolar.

En consecuencia, como objetivo general nos propusimos determinar el grado de relación entre violencia familiar y autoconcepto del educando. Para alcanzar tal propósito, se plantearon como objetivos específicos: diagnosticar el nivel de violencia familiar general en los padres de familia de los estudiantes. Identificar el nivel de violencia familiar en sus dimensiones psicológica, física, económica y sexual que experimentan los progenitores de familia. Evaluar el nivel de autoconcepto que presentan los educandos. Identificar el nivel de autoconcepto en sus dimensiones conductual, intelectual, físico, ansiedad, popularidad y felicidad-satisfacción, y finalmente, analizar la relación que existe entre las dimensiones de la violencia familiar y el autoconcepto de los educandos.

\section{MATERIAL Y MÉTODO}

Diseño de investigación: el diseño empleado fue descriptivo correlacional.

\section{Población, Muestra y Muestreo}

La población muestral estuvo constituida por 24 padres de familia (21 mamás y 3 papás) y 24 estudiantes (14 varones y 10 mujeres) de sexto grado de la I.E. N. ${ }^{\circ} 16309$ de la comunidad awajún Tayuntsa, distrito de Nieva, departamento de Amazonas, Perú.

\section{Métodos, técnicas e instrumentos de recolección de datos}

Se utilizó el método lógico deductivo, así como también el método lógico inductivo

Se empleó la Escala de Autoconcepto de Piers-Harris (PHCSCS). Piers y Harris (1969) a 24 estudiantes del sexto grado, conformado por 10 mujeres y 14 varones. Se evaluó por separado el nivel de violencia 
en el hogar y el autoconcepto del estudiante, para luego poder cruzar la información al encontrar el grado de correlación en ambas variables de estudio.

Así también, se aplicó el cuestionario de violencia familiar, elaborado por Jaramillo et al. (2014); teniendo por objetivo determinar el nivel de violencia familiar en los progenitores de familia. El cuestionario evaluó la violencia psicológica, física, económica y sexual. El instrumento presentó una valoración de escala Likert por pregunta de: siempre: 5; muy frecuente: 4; alguna ocasión: 3 , rara vez: 2 ; y nunca: 0

El instrumento sobre violencia familiar fue aplicado a los padres de familia constituida por 21 madres y 3 padres. Este criterio fue tomado, puesto que, la violencia se refleja en mayor proporción en la mujer en comparación a los varones.

La fiabilidad de la Escala de Autoconcepto de PiersHarris, presentó una consistencia interna de 0.88 a 0.93 . De igual manera, el cuestionario de violencia familiar, mantiene su consistencia interna a través del tiempo, con un Alfa de Cronbach inicial de 0,938, y un Alfa de Cronbach retest de 0,944 (Jaramillo et al., 2014). Las dimensiones al igual que la variable violencia familiar, mantiene su consistencia interna a través del tiempo.

\section{RESULTADOS}

Tabla 1

Distribución del nivel de violencia familiar y los tipos de violencia familiar en los padres de familia

\begin{tabular}{|c|c|c|c|}
\hline $\begin{array}{c}\text { Tipode } \\
\text { violencia } \\
\end{array}$ & Nivel & frecuencia & $\%$ \\
\hline \multirow{4}{*}{ Psicológica } & Sin violencia & 1 & $4 \%$ \\
\hline & Baja & 2 & $8 \%$ \\
\hline & Moderada & 17 & $71 \%$ \\
\hline & Alta & 4 & $17 \%$ \\
\hline \multirow{4}{*}{ Física } & Sin violencia & 3 & $13 \%$ \\
\hline & Baja & 6 & $25 \%$ \\
\hline & Moderada & 14 & $58 \%$ \\
\hline & Alta & 1 & $4 \%$ \\
\hline \multirow{4}{*}{ Económica } & Sin violencia & 0 & $0 \%$ \\
\hline & Baja & 7 & $29 \%$ \\
\hline & Moderada & 17 & $71 \%$ \\
\hline & Alta & 0 & $0 \%$ \\
\hline \multirow{4}{*}{ Sexual } & Sin violencia & 12 & $50 \%$ \\
\hline & Baja & 10 & $42 \%$ \\
\hline & Moderada & 2 & $8 \%$ \\
\hline & Alta & 0 & $0 \%$ \\
\hline \multirow{4}{*}{$\begin{array}{l}\text { Violencia } \\
\text { familiar }\end{array}$} & Sin violencia & 4 & $17 \%$ \\
\hline & Baja & 7 & $29 \%$ \\
\hline & Moderada & 11 & $46 \%$ \\
\hline & Alta & 2 & $8 \%$ \\
\hline \multicolumn{2}{|c|}{ Total } & 24 & $100 \%$ \\
\hline
\end{tabular}

La variable violencia familiar fue evaluada, según la muestra de estudio; es decir, en la tabla 1, se presenta los datos recolectados directamente de 24 padres de familia (21 madres y 3 padres) de los estudiantes del sexto grado de la I.E. 16309 de la comunidad awajún Tayuntsa. En tal sentido, en la tabla 1, se analizan los resultados obtenidos de la violencia familiar en general y en sus dimensiones de manera específica. Se puede evidenciar que los resultados de la violencia familiar en general fueron: $0 \%$ sin violencia, $38 \%$ baja, 63\% moderada, 0\% alta. Así también, según el tipo o dimensiones de violencia familiar se observan los siguientes resultados: para la violencia psicológica el 4\% sin violencia, $8 \%$ baja, $71 \%$ moderada, $17 \%$ alta, para la violencia física el $13 \%$ sin violencia, $25 \%$ baja, $58 \%$ moderada, $4 \%$ alta, violencia económica el $0 \%$ sin violencia, $29 \%$ baja, $71 \%$ moderada y el $0 \%$ alta, en la violencia sexual el $50 \%$ sin violencia, $42 \%$ baja, $8 \%$ moderada, $0 \%$ alta. Por lo que se evidencia en general, que la violencia familiar tiene una mayor proporción de nivel moderada y según el tipo de violencia para la psicológica, física y económica son de nivel moderada con mayor proporción y para la sexual entre sin violencia y violencia baja de este tipo.

Por otra parte, la variable autoconcepto fue evaluada en una muestra de 24 estudiantes (10 mujeres y 14 varones), a los que se suministró la Escala de Autoconcepto de Piers-Harris; sin embargo, en el tratamiento estadístico no se realizó el análisis según sexo, puesto que, solo se evaluó el nivel de violencia en el hogar del estudiante por separado y el autoconcepto del estudiante, para luego poder cruzar la información y encontrar el grado de correlación en las variables de estudio.

En tal sentido, en la tabla 2, se obtuvo los resultados de la variable autoconcepto como resultados generales se evidencia que el $46 \%$ de los estudiantes presentan un autoconcepto deficiente, el $38 \%$ pobre, el $17 \%$ moderado y el $0 \%$ alto. Así también, con respecto a las dimensiones del autoconcepto se encontró los siguientes resultados: para el conductual el $29 \%$ deficiente, $33 \%$ pobre, $38 \%$ moderado, $0 \%$ alto. 
$\underline{\text { Relación entre violencia familiar y autoconcepto del educando Mary Denisse Calvay Gomez, Ermerith Kiak Timias }}$

Tabla 2

Distribución del nivel de autoconcepto y los tipos de autoconcepto en los estudiantes

\begin{tabular}{|c|c|c|c|}
\hline $\begin{array}{c}\text { Tipode } \\
\text { A utoconcepto }\end{array}$ & Nivel & frecuencia & $\%$ \\
\hline \multirow{4}{*}{ Conductual } & Deficiente & 7 & $29 \%$ \\
\hline & Pobre & 8 & $33 \%$ \\
\hline & Moderado & 9 & $38 \%$ \\
\hline & Alto & 0 & $0 \%$ \\
\hline \multirow{4}{*}{ Intelectual } & Deficiente & 10 & $42 \%$ \\
\hline & Pobre & 9 & $38 \%$ \\
\hline & Moderado & 5 & $21 \%$ \\
\hline & A lto & 0 & $0 \%$ \\
\hline \multirow{4}{*}{ Físico } & Deficiente & 6 & $25 \%$ \\
\hline & Pobre & 10 & $42 \%$ \\
\hline & Moderado & 5 & $21 \%$ \\
\hline & Alto & 3 & $13 \%$ \\
\hline \multirow{4}{*}{ Ansiedad } & Deficiente & 2 & $8 \%$ \\
\hline & Pobre & 7 & $29 \%$ \\
\hline & Moderado & 13 & $54 \%$ \\
\hline & Alto & 2 & $8 \%$ \\
\hline \multirow{4}{*}{ Popularidad } & Deficiente & 11 & $46 \%$ \\
\hline & Pobre & 13 & $54 \%$ \\
\hline & Moderado & 0 & $0 \%$ \\
\hline & Alto & 0 & $0 \%$ \\
\hline \multirow{4}{*}{$\begin{array}{c}\text { felicidad } \\
\text { satisfacción }\end{array}$} & Deficiente & 13 & $54 \%$ \\
\hline & Pobre & 7 & $29 \%$ \\
\hline & Moderado & 4 & $17 \%$ \\
\hline & Alto & 0 & $0 \%$ \\
\hline \multirow{4}{*}{ A utoconcepto } & Deficiente & 10 & $42 \%$ \\
\hline & Pobre & 8 & $33 \%$ \\
\hline & Moderado & 4 & $17 \%$ \\
\hline & Alto & 2 & $8 \%$ \\
\hline \multicolumn{2}{|c|}{ Total } & 24 & $100 \%$ \\
\hline
\end{tabular}

Así también, para la dimensión intelectual el $42 \%$ fue deficiente, $38 \%$ pobre, $21 \%$ moderado, $0 \%$ alto; para el físico el $25 \%$ deficiente, $42 \%$ pobre, $21 \%$ moderado, $13 \%$ alto; para ansiedad el $8 \%$ deficiente, $29 \%$ pobre, $54 \%$ moderado, $8 \%$ alto; para popularidad el $46 \%$ deficiente, $54 \%$ pobre, $0 \%$ moderado, $0 \%$ alto; y para el de felicidadsatisfacción el $54 \%$ deficiente, $29 \%$ pobre, $17 \%$ moderado, $0 \%$ alto. Por lo general, el autoconcepto es de nivel deficiente a pobre con mayor proporción y según los tipos o dimensiones de autoconcepto, también es de nivel deficiente a pobre en mayor proporción.
Tabla 3

Correlaciones de Pearson bivariadas de los puntajes de la violencia familiar y dimensiones con el

\begin{tabular}{|c|c|c|c|c|c|c|}
\hline \multirow{6}{*}{ 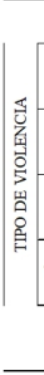 } & & Esta distico & Autoconcepto & $\begin{array}{c}\text { Significancia } \\
\text { (p-valor) }\end{array}$ & $\begin{array}{c}\text { Existe } \\
\text { Correlación }\end{array}$ & $\begin{array}{c}\text { Grado de } \\
\text { Correlación }\end{array}$ \\
\hline & $\begin{array}{c}\text { Violencia } \\
\text { psicológica }\end{array}$ & $\begin{array}{l}\text { Correlación de Pearson } \\
\text { Sig. (bilateral) } \\
\text { N }\end{array}$ & $\begin{array}{c}-0.527^{*} \\
0.028 \\
24.00\end{array}$ & $P<0.05$ & $\mathrm{Si}$ & $\begin{array}{c}\text { Moderada } \\
\text { inversa }\end{array}$ \\
\hline & Violencia fisica & $\begin{array}{l}\text { Correlación de Pearson } \\
\text { Sig. (bilateral) } \\
\text { N }\end{array}$ & $\begin{array}{l}-0.503^{*} \\
0.030 \\
24.00\end{array}$ & $\mathrm{P}<0.05$ & $\mathrm{Si}$ & $\begin{array}{c}\text { Moderada } \\
\text { inversa }\end{array}$ \\
\hline & $\begin{array}{c}\text { Violencia } \\
\text { económica }\end{array}$ & $\begin{array}{l}\text { Correlación de Pearson } \\
\text { Sig. (bilateral) } \\
\text { N }\end{array}$ & $\begin{array}{c}-0.493^{*} \\
0.032 \\
24.00\end{array}$ & $P<0.05$ & $\mathrm{Si}$ & $\begin{array}{c}\text { Moderada } \\
\text { inversa }\end{array}$ \\
\hline & Violencia sexual & $\begin{array}{l}\text { Correlación de Pearson } \\
\text { Sig. (bilateral) } \\
\text { N }\end{array}$ & $\begin{array}{c}-0.458^{*} \\
0.039 \\
24.00\end{array}$ & $\mathrm{P}<0.05$ & $\mathrm{Si}$ & $\begin{array}{c}\text { Moderada } \\
\text { inversa }\end{array}$ \\
\hline & $\begin{array}{l}\text { Viole ncia } \\
\text { familiar }\end{array}$ & $\begin{array}{l}\text { Corre lación de Pearson } \\
\text { Sig. (bilate ral) } \\
\text { N }\end{array}$ & $\begin{array}{l}-0.518^{\star} \\
0.030 \\
24.00\end{array}$ & $\mathrm{P}<0.05$ & Si & $\begin{array}{c}\text { Moderada } \\
\text { inversa }\end{array}$ \\
\hline
\end{tabular}

En la tabla 3, se puede evidenciar que existe una correlación moderada inversa o negativa entre la violencia familiar y dimensiones con el autoconcepto; es decir, para la violencia familiar en general, se obtuvo una correlación moderada inversa con el autoconcepto $(\mathrm{r}=-0.518, \mathrm{p}<0.05)$, así también sucede con las dimensiones de la variable violencia familiar $\mathrm{y}$ el autoconcepto: psicológica $(\mathrm{r} 1=-0.527, \mathrm{p}<0.05$, moderada inversa), física $(\mathrm{r} 2=-0.503, \mathrm{p}<0.05$, moderada inversa), económica ( $\mathrm{r} 3=-0.493, \mathrm{p}<0.05$, moderada inversa), sexual ( $\mathrm{r} 4=-0.458, \mathrm{p}<0.05$, moderada inversa) con el Autoconcepto.

\section{DISCUSIÓN}

Los resultados evidencian que existe una correlación negativa entre la violencia familiar y el autoconcepto $(\mathrm{r}=-0.518, \mathrm{p}<0.05)$, así también sucede con las dimensiones de la variable violencia familiar y el autoconcepto: psicológica $(\mathrm{r} 1=-0.527, \mathrm{p}<0.05$, moderada inversa), física $(\mathrm{r} 2=-0.503, \mathrm{p}<0.05$, moderada inversa), económica ( $\mathrm{r} 3=-0.493, \mathrm{p}<0.05$, moderada inversa), sexual $(\mathrm{r} 4=-0.458, \mathrm{p}<0.05$, moderada inversa) con el autoconcepto. Con tales resultados se puede predecir que a mayor violencia en menor el rendimiento académico de los estudiantes y a mayor autoconcepto, desde luego que el rendimiento será positivo; de manera semejante, Tuesta (2018) encontró que la violencia familiar es de nivel alto y los estudiantes provenientes de esos hogares presentaron deficiente rendimiento académico y sucede todo lo contrario cuando el nivel de violencia en el hogar es bajo. De igual modo, tales resultados relativamente se pueden comparar con Herrera, Al-Lal y Mohamed (2017) quienes encontraron correlaciones positivas de las calificaciones de los estudiantes con tres dimensiones del autoconcepto: académico, social y familiar evaluado. Por su parte, Benites y Montero (2019) confirman que en los estudiantes donde son víctimas de violencia intrafamiliar el mayor porcentaje de 
rendimiento académico se encuentra en proceso; sin embargo, donde no hay violencia los escolares presentan logro previsto. En síntesis, se concluye que el rendimiento escolar se relaciona positivamente con autoconcepto académico en escolares de educación primaria.

Así también, al evaluar los niveles de violencia familiar, los resultados evidencian un $17 \%$ sin violencia, $29 \%$ de violencia familiar baja, $64 \%$ moderada y el $8 \%$ considerada como alta; de igual forma sucede con sus dimensiones; así, por ejemplo, para la violencia psicológica el 71\% moderada y el $17 \%$ alta, para la violencia física el $58 \%$ moderada y $4 \%$ alta; la violencia económica presentó el $29 \%$ baja, $71 \%$ moderada y el $0 \%$ alta, en la violencia sexual el $50 \%$ sin violencia, $42 \%$ baja, $8 \%$ moderada. Estos resultados, son semejantes a los obtenidos por Silva (2017) quien halló que es alto el maltrato psicológico-emocional, ejercido por el hombre sobre la mujer, este acto al parecer es normal, ya que la mujer acepta que es el hombre el quien tiene el poder sobre la mujer. Así también, seguida de la violencia psicológica, se observa la violencia sexual, entre otras violencias ejercidas y sin embargo tales actos aún no son denunciados por las mujeres. También se rescata las apreciaciones de los estudiantes provenientes de los padres víctimas de violencia quienes afirman también sufrir las consecuencias de sus padres y que tales perturbaciones no les permite concentrarse del todo en sus clases.

Por su parte, Fresco, Arrom, C., Arrom, M. A., Ruoti, Arrom, C. M., Capurro (2018), encontraron que en el $71,8 \%$ (456) de los hogares, la población fue testigo de violencia psicológica, en hogares con bajos ingresos. De igual modo, el 26,9 \% (171 hogares) fueron espectadores de violencia física. Finalmente, los autores arriban a la conclusión que los elementos de vulnerabilidad socio-económica acompañan a mayoría de hogares con mujeres embarazadas; así también, los niños(as) y adolescentes son víctimas y testigos de violencia doméstica. En contraste, Flores y Tuesta (2018) encontraron que un $41 \%$ de los estudiantes expresan que en su casa si se preocupan por los resultados de sus calificaciones mientras que un $59 \%$ manifiestan que no, específicamente en las familias consideradas disfuncionales.

De igual manera a lo expuesto anteriormente, López (2017) corroboró que el $58 \%$ de los estudiantes que fueron víctimas de violencia familiar en sus hogares; el $39 \%$ indica que en su casa siempre existe violencia física; el $44 \%$ psicológica y el $37 \%$ verbal.

Por último, terminamos este capítulo, sincerando que los resultados al obtenerse en un solo contexto y en una sola medición, no pueden extrapolarse a toda la población; sin embargo, pueden dar orígenes a nuevas investigaciones y nuevos hallazgos en este campo de acción.

\section{CONCLUSIONES}

Existe una correlación moderada inversa o negativa entre la violencia familiar y el autoconcepto del educando del sexto grado en la Institución Educativa Primaria N. ${ }^{\circ} 16309$ de la comunidad Awajún Tayuntsa, del distrito de Nieva, $2017(\mathrm{R}=-0.518$, $\mathrm{P}=0.029<0.05$ ).

El nivel de violencia familiar es moderada-alta (54\%) en los padres de familia de los educandos del sexto grado en la Institución Educativa Primaria N. ${ }^{\circ} 16309$ de la comunidad Awajún Tayuntsa, del distrito de Nieva, 2017.

El nivel por tipo de violencia: psicológica es moderada-alta $(88 \%)$, física es moderada-alta (62\%), económica es moderada-alta $(71 \%)$ y sexual es bajamoderada $(50 \%)$ en los padres de familia de los educandos del sexto grado de la Institución Educativa Primaria N. ${ }^{\circ} 16309$ de la comunidad Awajún Tayuntsa, del distrito de Nieva, 2017.

El nivel de autoconcepto es deficiente-pobre (75\%) en el educando del sexto grado en la Institución Educativa Primaria N. ${ }^{\circ} 16309$ de la comunidad Awajún Tayuntsa, del distrito de Nieva, 2017.

El nivel por tipo de autoconcepto: conductual es deficiente-pobre $(62 \%)$, intelectual es deficientepobre $(80 \%)$, físico es deficiente-pobre $(67 \%)$, ansiedad es deficiente-pobre (37\%), popularidad es deficiente-pobre $(100 \%)$, felicidad-satisfacción es deficiente-pobre (84\%), en los educandos del sexto grado en la Institución Educativa Primaria N. ${ }^{\circ} 16309$ de la comunidad Awajún Tayuntsa, del distrito de Nieva, 2017.

Existe una correlación moderada inversa o negativa entre la los tipos de violencia familiar y el autoconcepto del educando del sexto grado en la Institución Educativa Primaria N. ${ }^{\circ} 16309$ de la comunidad Awajún Tayuntsa, del distrito de Nieva, 2017; [ $(\mathrm{R} 1=-0.527, \mathrm{P}=0.028<0.05), \quad(\mathrm{R} 2=-0.503$, $\mathrm{P}=0.030<0.05),(\mathrm{R} 3=-0.493, \mathrm{P}=0.032<0.05),(\mathrm{R} 4=-$ $0.458, \mathrm{P}=0.039<0.05)]$, respectivamente.

\section{REFERENCIAS BIBLIOGRÁFICAS}

Alcázar, L. y Ocampo, D. (2016). Consecuencias de la violencia doméstica contra la mujer en el progreso escolar de los niños y niñas del Perú. Documento de Investigación. GRADE.

Annerback, E. M., Wingren, G. y Gutafsson, P. A (2010). Prevalencia y características del abuso físico infantil en Suecia: los resultados forman una encuesta juvenil basada en la 
población. Acta Pediátrica, 99 (8), 1229-1236. https://bit.ly/3mD1TRv

Benites, R. y Montero, N. (2019). Violencia intrafamiliar y rendimiento académico en escolares, Huamachuco 2018. (Tesis de licenciatura; Universidad Nacional de Truji 1lo). https://bit.ly/34xyu4U

Bernal, F. y Gálvez, F. (2017). Ajuste escolar y rendimiento académico y su relación con la motivación y el autoconcepto. Análisis de diferencias en muestra chilena y española. Revista de Orientación Educacional, 31(60), 3-21. https://bit.ly/3ly3x7n

Dubowitz, H. y Benett, S. (2007). Abuso físico y abandono de niños. Lancet, 369 (9576), 18911899. https://doi.org/10.1016/S0140 6736(07

Flores, J. J. y Tuesta, N. (2018). Funcionalidad familiar y fracaso escolar en los alumnos de la institución educativa $N^{\circ} 1190$ Felipe Huamán Poma de Ayala - Chosica 2016. (Tesis de licenciatura; Universidad Nacional José Faustino Sánchez Carrión). https://bit.ly/2U8

Fresco, M. P., Arrom, C., Arrom, M. A., Ruoti, M., Arrom, C. M., Capurro, M. (2018). Hogares de madres embarazadas con niños, niñas y adolescentes testigos de violencia doméstica. Rev. Cient. Estud. Investig., 7(1), 92-100. 10.26885/rcei.7.1.92

Goñi, E. \& Fernández, A. (2008). El autoconcepto. En A. Goñi (Coord.). El autoconcepto físico: psicología y educación (pp. 23-57). Pirámide.

Herrera, L., Al-Lal, M. y Mohamed, L. (2017). Rendimiento escolar y autoconcepto en educación primaria. International Journal of Developmental and Educational Psychology INFAD Revista de Psicología, 2 (1), 315-326. https://bit.ly/3mHnVT6

INEI. Instituto Nacional de Estadística e Informática - Encuesta Demográfica y de Salud Familiar (2013). Violencia contra las mujeres, niñas y niños. Lima, Perú: INEI. https://goo.gl/nDBH

INEI. Instituto Nacional de Estadística e Informática (2017). Perú: indicadores de violencia familiar y sexual, 2000-2017. https://bit.ly/2u FnGCR

Jaramillo, J. et al. (2014). Construcción de una escala de violencia intrafamiliar, Cuenca Ecuador 2013. Revista de la Facultad de Ciencias Médicas, Universidad de Cuenca, 32(2), 30-39. https://bit.ly/3bVJ6g7

Jouriles, E. N., McDonald, R., Smith, A.M., y Heyman, R. E. (2008) Abuso infantil en el contexto de la violencia doméstica. Violenciay víctimas, 23(2), 221-235. https://bit.ly/ 3nHRVzA

López, M. B. (20|7). Presencia de violencia familiar en hogares de estudiantes de la I.E. Mariscal Castilla- 2016. (Tesis de licenciatura; Universidad Nacional del Centro del Perú). https://bit.ly/2RE0rSU

OMS. Organización Mundial de la Salud (2009). Las mujeres y la salud. Organización Mundial de la Salud. https://bit.ly/36EbNdu

Orna, O. (2013). Factores determinantes de la violencia familiar y sus implicancias: análisis de los estudios estadísticos sobre la Violencia Familiar en el distrito de San Juan de Lurigancho (Lima), Callao y otras ciudades del país. (Tesis de maestría; Universidad Nacional Mayor De San Marcos). https://goo. $\mathrm{gl} / \mathrm{Ms} 3 \mathrm{~m} 5$

Patró, R. y Limaña, R. (2005). Victimas de violencia familiar: Consecuencias psicológicas en hijos de mujeres maltratadas. Anales de psicología, 21(1), 11-17. https://bit.ly/3nIb2JN

Piers, E. V. y Harris, D. B. (1969). La escala de autoconcepto infantil Piers-Harris. Grabación del Consejero y Pruebas.

Silva, M. (2017). La violencia familiar (conyugal/pareja) en las ciudades de Cartagena y Barranquilla en el Caribe colombiano. Pensamiento Americano, 10(18), 159-176. https://bit.ly/2WCfwWM

Tuesta, G. L. (2018). Violencia familiar y su incidencia en el rendimiento académico de los estudiantes de la Institución Educativa $N^{\circ}$ 18040, Huancas, Amazonas, 2018. (Tesis de licenciatura; Universidad Nacional Toribio Rodríguez de Mendoza de Amazonas). https://bit.ly/3h7BWZ6 